\title{
Assessing the Association of Formulary Copayment Changes with Real-World Treatment Patterns in Patients with Rheumatoid Arthritis on Etanercept
}

\author{
Machaon Bonafede, PhD, MPH; Hafiz Oko-osi, MS, RPh; Mahdi Gharaibeh, PharmD, MS, PhD; \\ Janna Manjelievskaia, PhD, MPH; Lorena Lopez-Gonzalez, PhD, MA; David H. Collier, MD; \\ and Bradley S. Stolshek, PharmD
}

\begin{abstract}
BACKGROUND: Rheumatoid arthritis (RA) is a chronic disease that requires long-term treatment to improve or maintain stable disease activity. Tumor necrosis factor inhibitors (TNFi), a class of biologic disease-modifying antirheumatic drugs (bDMARD), are effective at treating symptoms and inhibiting joint progression. Although treatment changes are not recommended in patients with stable disease, health plans have recently enacted formulary changes with higher copayments that could disrupt patient access to TNFis.

OBJECTIVE: To assess the association of formulary copayment changes with real-world treatment patterns, treatment effectiveness, and health care costs among bDMARD-naive patients with RA receiving the TNFi etanercept.
\end{abstract}

METHODS: This retrospective observational cohort analysis used the IBM Watson Health MarketScan Commercial Claims and Encounters Database. Adult patients with RA with 6 months of stable etanercept use (no refill gap $\geq 45$ days) from January 1, 2013, through December 31, 2015, were selected and the index date was set to the first fill date after the stable-use period. Average etanercept copayment was calculated at the drug-plan level. Copayment change was defined as a monthly increase of at least $\$ 40$ to account for copayment changes attributable to etanercept wholesale acquisition costs between 2014 and 2015. This amount also corresponded to the 90th percentile of average plan-level changes in etanercept copayments in the database, representing an average change in copayment by a payer. Patients were followed $\geq 12$ months before and after the index date to track etanercept treatment changes and $\geq 12$ months after a treatment change to track costs after etanercept copayment changes. Etanercept persistence, bDMARD switching, refill gaps, and treatment effectiveness (using a validated effectiveness algorithm) were described for patients with or without copayment change during the 12 months post-index or postchange. We also assessed the mean total of all-cause and RA-related expenditure during the 12-month post-index (or postchange) period.

RESULTS: 1,970 stable patients met study inclusion criteria (mean [standard deviation] age: 50.3 [9.5] years; $77.8 \%$ female) and were evaluated. Of these, $133(6.8 \%)$ patients had a copayment change $\geq \$ 40$ during follow-up. Overall, most patients $(60.3 \%)$ persisted on etanercept for the 12-month follow-up period, while $13.0 \%$ switched from etanercept, and $8.1 \%$ discontinued (refill gap of $\geq 45$ days). Nearly half $(48.0 \%)$ of all patients were considered effectively treated according to a validated algorithm. Compared with patients without a copayment change, those with a copayment change were more likely to switch biologics $(19.5 \%$ vs. $12.6 \% ; P=0.021)$. Although statistical significance was not reached, patients with a copayment change were less likely to be persistent $(54.1 \%$ vs. $60.7 \% ; P=0.135)$, and less likely to be effectively treated $(42.1 \%$ vs. $48.4 \% ; P=0.161$ ) than patients without a copayment change. All-cause and RA-related expenditures at baseline and post-copayment change were similar between patients with and without a copayment change.
CONCLUSIONS: Changing formulary copayment of etanercept was associated with higher switching without difference in costs or health care utilization between copayment and no copayment change groups.

\section{J Manag Care Spec Pharm. 2020;26(2):211-20}

Copyright $\odot 2020$, Academy of Managed Care Pharmacy. All rights reserved.

\section{What is already known about this subject}

Although treatment changes are not recommended in patients with stable disease, health plans have recently enacted formulary copayment changes with higher tier drugs having higher copayments, which could disrupt patient access to tumor necrosis factor inhibitors

Evidence suggests that copayment increases and the associated nonmedical biologic switching are detrimental to patient health and increase health care resource utilization and costs among patients with rheumatoid arthritis (RA).

\section{What this study adds}

This study furthers our understanding of formulary copayment increases and real-world treatment patterns and treatment effectiveness among patients with RA who are naive to biologic diseasemodifying antirheumatic drugs and are receiving etanercept.

Changing copayments of etanercept was associated with higher nonmedical switching to another RA medication. Validation in a larger study is recommended.

$R$ heumatoid arthritis (RA) is a chronic disease that requires long-term treatment to improve or maintain stable disease activity. Affecting approximately 1.3 million adults in the United States, RA is characterized by persistent inflammation in joints, which damages the surrounding cartilage and bone and negatively impacts physical health and patient functionality because of pain, swelling, and stiffness. ${ }^{1}$ When unmanaged, RA leads to progressive joint damage resulting in physical impairment and long-term disability.

Effective treatment of RA symptoms with disease-modifying antirheumatic drugs (DMARDs) has been shown to increase 
productivity at work and home and to improve health-related quality of life. ${ }^{2,3}$ Furthermore, the addition of a biologic DMARD (bDMARD) or a targeted synthetic DMARD to the treatment regimen is recommended for patients with RA who have moderate or high disease activity despite monotherapy with a conventional synthetic DMARD. ${ }^{4}$ Tumor necrosis factor inhibitors (TNFis), a class of targeted immune modulators, are effective at treating RA symptoms and inhibiting joint progression. ${ }^{5}$ TNFis are indicated for the treatment of moderate to severe RA, with etanercept being one of the most commonly prescribed. ${ }^{6,7}$

Nonpersistence on biologic therapy may occur because of medical reasons (such as inadequate response or medication intolerance) or nonmedical reasons, defined as a reason unrelated to clinical efficacy or tolerability. ${ }^{8-10}$ Nonmedical reasons may include economic reasons, such as increased copay, change of insurance, job loss, or other economic factors that limit the affordability of a medication. ${ }^{11}$ Although treatment changes are not recommended in patients with stable disease, formulary changes to health plans, such as increasing copayments or categorizing therapeutic agents in higher tiers, could disrupt patient access to TNFi therapy. ${ }^{4}$ Use of drugs in higher formulary tiers, often with higher copayment cost, may require prior approval from insurance providers and could drive patients to switch therapies for reasons unrelated to their RA management. Evidence suggests that formulary copayment changes and the associated nonmedical biologic switching are not only detrimental to patient health but also increase health care resource utilization and costs among patients with RA. ${ }^{12-15}$ Patients who switched for nonmedical reasons had increased disease flares, poorer disease control, had 14\% more RA-related outpatient visits, and 29\% more rheumatologist visits than patient persistent on their TNFi therapy. ${ }^{11,13,14}$ Furthermore, nonmedical switchers incurred significantly higher all-cause medical costs ( $\$ 4,557$ vs. $\$ 3,310 ; P=0.0011)$ and total costs $(\$ 21,996$ vs. $\$ 17,379 ; P<0.001)$ than patients persistent on their TNFi therapy. ${ }^{12}$

This study sought to understand the effect of formulary copayment changes on real-world treatment patterns, treatment effectiveness, and health care costs among bDMARDnaive patients with RA receiving etanercept.

\section{Methods}

\section{Study Design}

We performed a retrospective analysis of data from IBM Watson Health MarketScan Commercial Claims and Encounters Database, a large U.S. employer-based claims database that includes claims from approximately 137.6 million employees and their dependents. Data were from January 1, 2012, through December 31, 2016. This study used de-identified database records, which are fully compliant with the U.S. Health Insurance Portability and Accountability Act (HIPAA); this compliance with HIPAA regulation meant that patient consent and institutional review board approval to conduct this study were not necessary.

Patients were grouped according to whether they were in a plan that experienced an etanercept copayment change or not. Copayment change was defined as a monthly increase of at least $\$ 40$ to account for copayment changes attributable to difference in etanercept wholesale acquisition costs between 2014 and 2015. The $\$ 40$ increase cutoff was derived by assessing copayments across 3 - and 4-tiered formularies and calculating expected copayment changes based on etanercept wholesale acquisition cost changes. The cutoff also corresponds with the 90th percentile copayment amount among the eligible cohort.

The study design is outlined in Appendix A (available in online article). Study periods were defined as follows: Index date was the first fill date after a patient's 6-month stable etanercept use period (i.e., no refill gap $\geq 45$ days). Baseline period was defined as the 12 months preceding the index date, which included 6 months of no TNFi use (to establish a bDMARDnaive population), followed by the patient's 6-month stable use period. Demographic characteristics were captured on the index date. Clinical characteristics were determined from claims data during the 12-month baseline period. Post-index period was defined as the period following the index date (up to 12 months) and was used for assessment of etanercept treatment changes. Postchange period was defined as the 12 months after treatment changes for patients who were nonpersistent on etanercept (persistence was defined as having no refill gap $\geq 45$ days and no switching therapy). Outcome measures were assessed post-index in the persistence group and postchange in the treatment change group. To ensure cost assessments were over a consistent duration, participants were required to have a continuous plan enrollment for $\geq 12$ months post-index date or for $\geq 12$ months postchange. Post-index periods were equal to the postchange period for persistent patients (Appendix A).

\section{Study Population}

Inclusion Criteria. Patient selection is described in Appendix B (available in online article). Adults (18-64 years) with RA who had at least 6 months of continuous etanercept use from January 1, 2013, through December 31, 2015, no pharmacy or medical claims for any TNFi during the 6 months before the 6-month stable etanercept use period were evaluated.

Exclusion Criteria. Patients were excluded if they had a nondiagnostic medical claim with an International Classification of Diseases, Ninth/Tenth Revision, Clinical Modification diagnosis code for juvenile idiopathic arthritis, psoriasis, psoriatic arthritis, ankylosing spondylitis, Crohn disease, or ulcerative colitis at any time during the study period. 


\section{Assessing the Association of Formulary Copayment Changes with Real-World Treatment Patterns in Patients with Rheumatoid Arthritis on Etanercept}

\section{TABLE 1 Demographic and Clinical Characteristics for Patients with 6-Month Stable Etanercept Use}

\begin{tabular}{|c|c|c|c|c|c|c|}
\hline \multirow[b]{2}{*}{ Mean (SD) age, years } & \multicolumn{2}{|c|}{$\begin{array}{l}\text { No Copayment Change } \\
\qquad(\mathrm{n}=1,837)\end{array}$} & \multicolumn{2}{|c|}{$\begin{array}{l}\text { Copayment Change } \\
(n=133)\end{array}$} & \multicolumn{2}{|c|}{$\begin{array}{c}\text { All } \\
(\mathrm{N}=1,970)\end{array}$} \\
\hline & 50.4 & $(9.5)$ & 50.0 & $(9.7)$ & 50.3 & $(9.5)$ \\
\hline \multicolumn{7}{|l|}{ Age category, \% (n) } \\
\hline $18-34$ & 7.8 & (143) & 8.3 & (11) & 7.8 & (154) \\
\hline $35-44$ & 17.0 & (313) & 16.5 & (22) & 17.0 & (335) \\
\hline $45-54$ & 35.4 & $(651)$ & 37.6 & (50) & 35.6 & $(701)$ \\
\hline $55-64$ & 39.7 & $(730)$ & 37.6 & $(50)$ & 39.6 & $(780)$ \\
\hline Female, \% (n) & 77.5 & $(1,424)$ & 82.0 & $(109)$ & 77.8 & $(1,533)$ \\
\hline \multicolumn{7}{|l|}{ Insurance plan type, \% (n) } \\
\hline Comprehensive/indemnity & 4.7 & (86) & 0.0 & $(0)$ & 4.4 & $(86)$ \\
\hline EPO/PPO & 59.0 & $(1,084)$ & 66.9 & (89) & 59.5 & $(1,173)$ \\
\hline POS/POS with capitation & 5.9 & (109) & 10.5 & (14) & 6.2 & (123) \\
\hline $\mathrm{HMO}$ & 11.2 & (206) & 9.8 & (13) & 11.1 & (219) \\
\hline CDHP/HDHP & 17.9 & (328) & 12.8 & (17) & 17.5 & $(345)$ \\
\hline Unknown & 1.3 & $(24)$ & 0.0 & $(0)$ & 1.2 & $(24)$ \\
\hline \multicolumn{7}{|l|}{ Index year, \% (n) } \\
\hline 2013 & 26.6 & $(488)$ & 47.4 & (63) & 28.0 & $(551)$ \\
\hline 2014 & 43.0 & $(789)$ & 28.6 & (38) & 42.0 & $(827)$ \\
\hline 2015 & 30.4 & $(559)$ & 24.1 & (32) & 30.0 & $(591)$ \\
\hline 2016 & 0.0 & (1) & 0.0 & $(0)$ & 0.0 & (1) \\
\hline Deyo-Charlson Comorbidity Index, mean (SD) & 1.4 & $(0.9)$ & 1.5 & $(0.9)$ & 1.4 & $(0.9)$ \\
\hline \multicolumn{7}{|l|}{ Comorbid conditions, \% (n) } \\
\hline \multicolumn{7}{|l|}{ Chronic respiratory/pulmonary conditions } \\
\hline Asthma & 16.7 & $(307)$ & 15.8 & $(21)$ & 16.6 & $(328)$ \\
\hline Chronic obstructive pulmonary disease & 4.0 & $(74)$ & 4.5 & (6) & 4.1 & $(80)$ \\
\hline Bronchiolitis obliterans organizing pneumonia & 0.2 & (4) & 0.0 & (0) & 0.2 & (4) \\
\hline Pulmonary fibrosis/interstitial lung disease & 2.3 & (43) & 0.0 & (0) & 2.2 & (43) \\
\hline Pulmonary embolism & 0.3 & (6) & 0.8 & (1) & 0.4 & $(7)$ \\
\hline \multicolumn{7}{|l|}{ Cardiovascular disease } \\
\hline Congestive heart failure & 0.8 & (14) & 0.8 & (1) & 0.8 & (15) \\
\hline Coronary heart disease with or without old MI & 4.6 & $(84)$ & 6.8 & (9) & 4.7 & (93) \\
\hline Ischemic stroke/transient ischemic attack & 0.8 & $(14)$ & 0.0 & $(0)$ & 0.7 & $(14)$ \\
\hline Peripheral artery disease & 2.1 & (38) & 1.5 & (2) & 2.0 & $(40)$ \\
\hline Ventricular arrhythmia & 0.5 & (10) & 0.8 & (1) & 0.6 & (11) \\
\hline Depression & 12.0 & $(220)$ & 5.3 & $(7)$ & 11.5 & $(227)$ \\
\hline Diabetes & 10.2 & (188) & 13.5 & (18) & 10.5 & (206) \\
\hline Fibromyalgia & 11.8 & $(217)$ & 17.3 & (23) & 12.2 & $(240)$ \\
\hline Hypertension & 30.0 & $(551)$ & 39.8 & (53) & 30.7 & $(604)$ \\
\hline Serious infection ${ }^{\mathrm{a}}$ & 5.7 & (104) & 3.0 & (4) & 5.5 & (108) \\
\hline \multicolumn{7}{|l|}{ Concomitant medication use, \% (n) } \\
\hline Conventional DMARDs & 80.4 & $(1,477)$ & 84.2 & (112) & 80.7 & $(1,589)$ \\
\hline Glucocorticoids & 73.8 & $(1,356)$ & 77.4 & (103) & 74.1 & $(1,459)$ \\
\hline
\end{tabular}

ancludes inpatient infection or intravenous antibiotic use.

$C D H P=$ consumer-driven health plan; DMARD = disease-modifying antirheumatic drug; $E P O=$ exclusive provider organization; HDHP=high-deductible health plan;

$H M O=$ health maintenance organization; $M I=$ myocardial infarction; $P O S=$ point of service; $P P O=$ preferred provider organization; $S D=$ standard deviation.

\section{Outcomes}

Etanercept treatment patterns within the post-index period were assessed. Treatment patterns were defined as follows: persistence, when no treatment modifications or refill gaps ( $\geq 45$ days) occurred during the post-index period, and nonpersistence, when a refill gap $\geq 45$ days or biologic switch from etanercept occurred during the post-index period. Nonpersistent patients were further categorized into the following mutually exclusive categories: switch with a refill gap for etanercept, switch without a refill gap for etanercept, etanercept restart after a gap in treatment, and etanercept discontinuation.

A validated claims-based effectiveness algorithm that uses administrative data as a proxy for clinical response (as measured by the Disease Activity Score in 28 joints) was used to 


\begin{tabular}{|c|c|c|c|}
\hline $\begin{array}{l}\text { Treatr } \\
\text { Chan } \\
\text { (N=1 }\end{array}$ & $\begin{array}{l}\text { nent Pattern } \\
\text { ge in Stable } \\
970 \text { ) }\end{array}$ & $\begin{array}{l}\text { After Cop } \\
\text { anercept }\end{array}$ & $\begin{array}{l}\text { nent } \\
\text { ers }\end{array}$ \\
\hline & $\begin{array}{c}\text { No Copayment } \\
\text { Change }\end{array}$ & $\begin{array}{l}\text { Copayment } \\
\text { Change }\end{array}$ & $P$ Value \\
\hline Stable etanercept use, \% (n) & $93.2(1,837)$ & $6.8(133)$ & - \\
\hline Persistence, \% (n) & $60.7(1,115)$ & $54.1 \quad(72)$ & 0.135 \\
\hline Nonpersistence, \% (n) & $39.3 \quad(722)$ & $45.9 \quad(61)$ & - \\
\hline Refill gap & $26.7 \quad(491)$ & $26.3 \quad(35)$ & 0.917 \\
\hline Restart (those with gap) & $70.3 \quad(345)$ & $60.0 \quad(21)$ & 0.202 \\
\hline Discontinuation $^{\mathrm{a}}$ & $7.9 \quad(146)$ & $10.5 \quad(14)$ & 0.293 \\
\hline Switch & $12.6 \quad(231)$ & $19.5 \quad(26)$ & 0.021 \\
\hline Prior refill gap & $3.4 \quad(62)$ & $3.8 \quad(5)$ & 0.813 \\
\hline No prior refill gap & $9.2 \quad(169)$ & $15.8 \quad(21)$ & 0.013 \\
\hline $\begin{array}{l}\text { Any refill gap } \\
\text { (with or without switch) }\end{array}$ & $30.1 \quad(553)$ & $30.1 \quad(40)$ & 0.995 \\
\hline Effectively treated & $48.4 \quad(889)$ & $42.1 \quad(56)$ & 0.161 \\
\hline
\end{tabular}

describe effective or ineffective RA control during the 12-month post-index (or postchange) period. ${ }^{16}$ Patients who satisfied all 6 of the following algorithm criteria for the 12 months after the switch date were considered effectively treated:

1. Adherence (medication possession ratio of $\geq 80 \%$ )

2. No increase in dose or frequency once stable dose is achieved (post-index); no increase in dose or frequency after switching (postchange)

3. No switch or addition of new biologic

4. No addition of a new nonbiologic DMARD

5. No more than 1 intra-articular glucocorticoid injection

6. No new (or increased dose of) oral glucocorticoids

Additionally, we assessed the mean total all-cause and RA-related expenditures (outpatient and inpatient costs, and outpatient prescription costs) during the 12 -month post-index (or postchange) period (patient and plan). Health care costs were attributable to RA if there was a nondiagnostic medical claim with RA in any position on an outpatient claim. The whole hospitalization cost was attributable to RA if RA was the primary discharge diagnosis of the hospitalization. All dollar estimates were inflated to 2016 dollars using the medical care component of the Consumer Price Index.

\section{Statistical Considerations}

Descriptive statistics were used to describe outcomes of patients grouped by having a formulary copayment change or not. Fisher's exact test was used for categorical comparisons. A chi-square test was used to test for differences in categorical variables. Multivariable regression analysis was used to assess the relationship between formulary copayment changes and treatment outcomes and health care costs, adjusting for baseline demographic and clinical characteristics (age, index year,

\begin{tabular}{l|c|c|c}
\hline TABLE 3 & $\begin{array}{l}\text { Association Between Copayment } \\
\text { Change and Treatment Patterns After } \\
\text { Multivariable Adjustment }\end{array}$ \\
\hline & \multicolumn{3}{c}{ Effect of Copayment Change } \\
\cline { 2 - 4 } Outcome & Odds Ratio & P Value & $95 \%$ CI \\
\hline Nonpersistence & 1.255 & 0.231 & $(0.865-1.821)$ \\
\hline Biologic switch & 1.885 & 0.009 & $(1.170-3.039)$ \\
\hline Not effectively treated & 1.443 & 0.050 & $(1.000-2.082)$ \\
\hline Note: Variables used for adjustment include effectively treated in stable period; \\
baseline MTX use; baseline non-MTX conventional disease-modifying anti- \\
rheumatic drug use; Deyo-Charlson Comorbidity Index; baseline number of \\
emergency room visits; baseline number of rheumatologist office visits; health plan \\
type; age; index year; geographical region (South, North East, North Central, and \\
West); and baseline diagnoses of asthma, cardiovascular disease, fibromyalgia, \\
hypertension, and malignancy. A claims-based effectiveness algorithm (Curtis et al. \\
2011) was used to assess rheumatoid arthritis control during the 12-month post- \\
index (or postchange) period. ${ }^{16}$ \\
CI=confidence interval; MTX=methotrexate.
\end{tabular}

insurance plan type, geographical region, and baseline diagnosis [asthma, depression, diabetes, hypertension, infection, osteoarthritis, and osteoporosis]). The significance threshold was set at $P<0.05$.

\section{Results}

Overall, 1,970 patients with 6 months stable etanercept use met the study inclusion and exclusion criteria and were evaluated (Table 1). The mean patient age was 50.3 years. Patients were grouped according to formulary copayment change: no copayment change group $(n=1,837)$ and copayment change group $(n=133)$. Except for the index year, demographics and clinical characteristics were similar between groups. Most were female $(77.8 \%), \geq 45$ years $(75.2 \%)$, and receiving conventional DMARD $(80.7 \%)$ or glucocorticoid (74.1\%) concomitant medication. Overall, the mean copayment increase was $\$ 68.53$ for patients in the copayment change group and a decrease of $\$ 4.48$ for patients in the no copayment change group.

\section{Treatment Patterns}

Across all patients, $60.3 \%(1,187 / 1,970)$ were categorized as persistent etanercept users during the 12 -month post-index period (Table 2). Of the 783 nonpersistent patients, 32.8\% switched from etanercept treatment and $46.7 \%$ had a refill gap but subsequently restarted etanercept treatment. Overall, one fifth of nonpersistent patients discontinued etanercept treatment without switching or restarting treatment.

A larger proportion of patients in the copayment change group switched treatments than those in the no copayment change group ( $19.5 \%$ vs. $12.6 \%$; $P=0.021)$. Although statistical significance was not reached when compared with the no copayment change group, patients with copayment change tended to show higher rates of discontinuation $(10.5 \%$ vs. $7.9 \% ; P=0.293)$ and lower rates of persistence $(54.1 \%$ vs. $60.7 \% ; P=0.135)$. 


\section{FIGURE 1 Total All-Cause and RA-Related Costs at Baseline and Postformulary Change in Patients with and} Without a Copayment Change

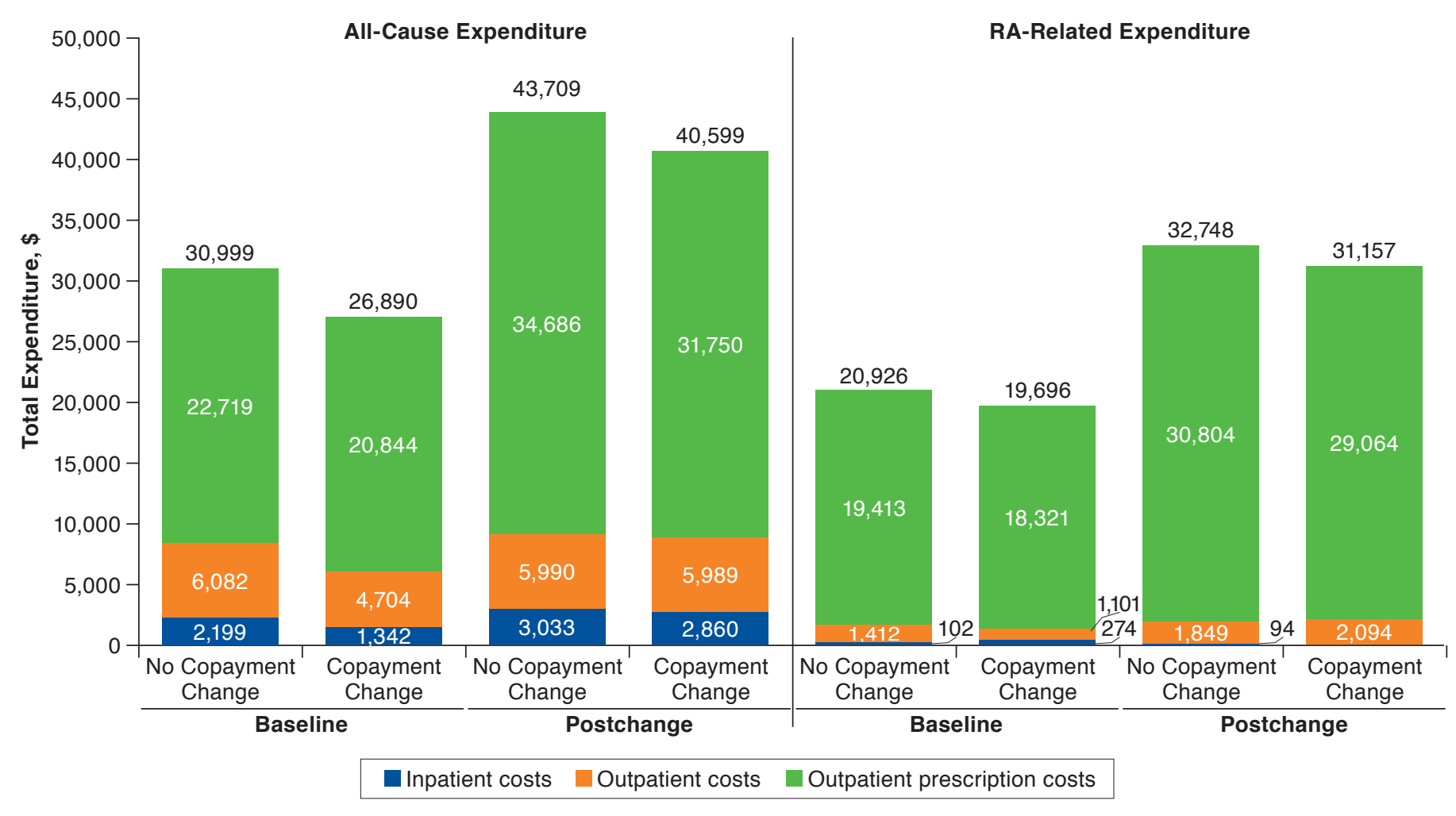

Note: Bar totals may be different due to rounding.

$\mathrm{RA}=$ rheumatoid arthritis

\section{Treatment Effectiveness}

Across all patients, 65.7\% (1,294/1,970) satisfied all 6 treatment effectiveness algorithm criteria during the 12-month post-index period. A larger proportion of persistent patients compared with nonpersistent patients were effectively treated (73.7\% vs. $53.5 \%)$. Although statistical significance was not reached when compared with the no copayment change group, patients with copayment change tended to have lower rates of effective treatment (42.1\% vs. $48.4 \%$; $P=0.161$; Table 2).

\section{Association Between the Formulary Copayment Change Group and Switching and Treatment Effectiveness}

After controlling for baseline demographics and clinical characteristics, patients with a copayment change had 1.88 higher odds of switching biologic agents during the post-index period than patients without a copayment change $(P=0.009)$. Additionally, patients with a copayment change had 1.44 higher odds of not being effectively treated during the postindex period, measured using a validated algorithm, than patients without a copayment change $(P=0.05)$. The odds of persistence with index therapy was similar regardless of copayment change status ( $P=0.231$; Table 3$)$.

\section{Costs}

The mean all-cause and RA-related expenditures at baseline and post-copayment change were similar between groups (Figure 1). Furthermore, after adjusting for potential confounders, multivariable analyses showed that total all-cause and total RA-related costs during the post-index period remained similar between patients regardless of copayment change (Table 4).

\section{Discussion}

Our data add to the growing evidence that formulary copayment changes are associated with nonmedical biologic switching, which may be detrimental to patient health among patients with RA. ${ }^{12-15}$ In summary, our analysis found that copayment changes were associated with higher rates of switching and lower rates of being effectively treated, without differences in costs. Consistent with previous findings that treatment persistence is associated with improvements in RA disease 


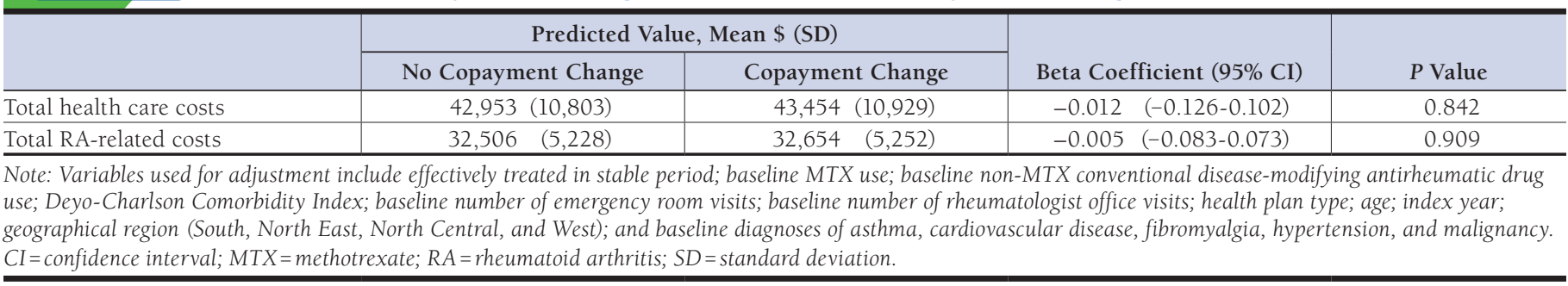

activity and disability, multivariable analyses demonstrated that patients without copayment change were more likely to be effectively treated according to the validated effectiveness algorithm. ${ }^{12-15}$ Discontinuation of anti-TNF therapy for nonmedical reasons has been shown to result in significantly worse clinical outcomes and increased health care resource utilization. ${ }^{12-15}$ In contrast to our findings, previous studies have shown that patients who switched to another DMARD for no apparent medical reason other than to lower out-of-pocket costs used significantly more health care services as compared with those who remained on their index drug. Specifically, those who switched treatment incurred $42 \%$ more emergency department visits, 29\% more rheumatologist visits, and 12\% more outpatient visits as compared with those who remained on index therapy. Additionally, patients switching for nonmedical reasons were more likely to discontinue their new DMARD in the 6-month follow-up period. ${ }^{12}$ Furthermore, patients who switched or discontinued TNFi treatment were at a significantly greater risk of flares than patients who continued TNFi treatment, as well as at an increased risk of emergency department visits and more repeat emergency department visits. ${ }^{13}$ Here, we demonstrated higher rates of switching without an increase in costs or health care utilization. This difference may reflect our cohort that was restricted to patients with 6 months stable etanercept use during the pre-index period and may represent a more homogenous population of stably treated patients than previous studies, ensuring our outcomes are not influenced by early nonpersistence.

Overall, rate of persistence observed in our study was higher than persistence rates in similar claims-based studies. ${ }^{17,18}$ The more homogenous and stable population may explain these differences, as TNFi switching usually occurs following 3 months of failed treatment. ${ }^{4}$ Previously, nonmedical switching of TNFi treatment has been shown to be associated with an increase in side effects and lack of efficacy that led to treatment adjustments and increased health care resource utilization. ${ }^{15}$ Furthermore, Liu et al. (2015) showed that patients who switched following a payer formulary change and for no apparent medical reason were found to incur significantly higher all-cause medical costs and total costs during follow-up than those maintaining index therapy. ${ }^{12}$ However, in contrast to these reports, and counterintuitively, we show that despite higher formulary copayments, total health care and RA-related costs remained similar, suggesting no increase in health care resource utilization. The small proportion of our cohort (6.8\%) who had a formulary copayment change may indicate that the study was insufficiently powered to detect the difference. Alternatively, our analysis required that patients have 6 months of stable etanercept use as an inclusion criterion, which would exclude patients with shorter treatment durations after initiation; shorter treatment duration can be associated with lower costs, as therapy costs comprise the majority of health care costs in patients with RA. ${ }^{19}$

\section{Strengths and Limitations}

Our study had several strengths, including the use of a large, prescription coverage-based retrospective cohort. We determined new incident use of etanercept by identifying no previous use of bDMARD in the year before index. Furthermore, the requirement of 6-month stable etanercept use means our population was more stable and homogenous than similar claimsbased studies without this criterion. However, further study is needed to determine if changes in coverage force more patients on a TNFi to switch treatment or have longer than expected refill gaps in therapy and if treatment changes are associated with changes in treatment effectiveness and health care costs.

Our study was subject to some limitations, including those inherent in the analysis of administrative claims data. The study was limited to individuals with 24-month continuous commercial health coverage between the ages of 18 and 64 years. As such, results of this analysis may not be generalizable to bDMARD-naive patients with RA $>65$ years or those with other types of insurance, those with intermittent health care coverage, or those without health insurance coverage.

Our definition of copayment change was empirically derived based on a plan-level analysis of copayment changes from year to year. Despite the strength of the large population size and real-world data, only a small proportion of our cohort (6.8\%) 


\section{Assessing the Association of Formulary Copayment Changes with Real-World Treatment Patterns in Patients with Rheumatoid Arthritis on Etanercept}

had a copayment change of $\geq \$ 40$, which negatively impacts detectable differences for our comparisons. However, as the copayment cutoff corresponded with the 90th percentile copayment amount among the eligible cohort, it should be expected that less than $10 \%$ would have had a copayment change.

There is no single gold standard for adjusting health expenditures for inflation. Here, all dollar estimates were inflated to 2016 dollars using the medical care component of the Consumer Price Index, which may have led to overestimates of medical expenditures compared with adjusting with the Personal Health Care price or Personal Consumption Expenditures health component price index. ${ }^{20}$ However, as there was no increase in health care resource utilization between those with and without copayment change, this finding is likely to stand regardless of index used.

\section{Conclusions}

This claims-based analysis of commercially insured patients in the United States showed that changing formulary status of etanercept was associated with higher switching that negatively impacted persistence and treatment effectiveness without differences in costs between no copayment change and copayment change groups. Further evaluation in a larger cohort of patients with a copayment change is recommended to validate these findings.

\section{Authors}

MACHAON BONAFEDE, PhD, MPH; JANNA MANJELIEVSKAIA $\mathrm{PhD}, \mathrm{MPH}$; and LORENA LOPEZ-GONZALEZ, PhD, MA, IBM Watson Health, Cambridge, Massachusetts. HAFIZ OKO-OSI, MS, RPh; MAHDI GHARAIBEH, PharmD, MS, PhD; DAVID H. COLLIER, MD; and BRADLEY S. STOLSHEK, PharmD, Amgen, Thousand Oaks, California.

AUTHOR CORRESPONDENCE: Machaon Bonafede, PhD, MPH, IBM Watson Health, 131 Pickpocket Rd., Brentwood, NH 03833. Tel.: 603.775.3141; E-mail: mbonafed@us.ibm.com.

\section{ACKNOWLEDGMENTS}

Medical writing support was provided by Jonathan Plumb, PhD, of Fishawack Communications, which was funded by Amgen.

\section{REFERENCES}

1. National Institutes of Health. Rheumatoid arthritis. November 12, 2019. Reviewed September 2013. Available at: https://ghr.nlm.nih.gov/condition/ rheumatoid-arthritis\#statistics. Accessed November 16, 2019.

2. Hone D, Cheng A, Watson C, et al. Impact of etanercept on work and activity impairment in employed moderate to severe rheumatoid arthritis patients in the United States. Arthritis Care Res (Hoboken). 2013;65(10):1564-72.

3. Strand V, Sharp V, Koenig AS, et al. Comparison of health-related quality of life in rheumatoid arthritis, psoriatic arthritis and psoriasis and effects of etanercept treatment. Ann Rheum Dis. 2012;71(7):1143-50.

4. Singh JA, Saag KG, Bridges SL Jr, et al. 2015 American College of Rheumatology guideline for the treatment of rheumatoid arthritis. Arthritis Rheumatol. 2016;68(1):1-26.

5. Guo Q, Wang Y, Xu D, Nossent J, Pavlos NJ, Xu J. Rheumatoid arthritis: pathological mechanisms and modern pharmacologic therapies. Bone Res. 2018;6:15

6. Lethaby A, Lopez-Olivo MA, Maxwell L, Burls A, Tugwell P, Wells GA Etanercept for the treatment of rheumatoid arthritis. Cochrane Database Syst Rev. 2013;(5):CD004525.

7. Calip GS, Adimadhyam S, Xing S, Rincon JC, Lee WJ, Anguiano RH Medication adherence and persistence over time with self-administered TNF-alpha inhibitors among young adult, middle-aged, and older patients with rheumatologic conditions. Semin Arthritis Rheum. 2017;47(2):157-64.

8. Gómez-Reino JJ, Rodríguez-Lozano C, Campos-Fernández C, Montoro M, Descalzo MÁ, Carmona L; on behalf of the BIOBADESER 2.0 Study Group. Change in the discontinuation pattern of tumour necrosis factor antagonists in rheumatoid arthritis over 10 years: data from the Spanish registry BIOBADASER 2.0. Ann Rheum Dis. 2012;71(3):382-85.

9. Markenson JA, Gibofsky A, Palmer WR, et al. Persistence with anti-tumor necrosis factor therapies in patients with rheumatoid arthritis: observations from the RADIUS registry. J Rheumatol. 2011;38(7):1273-81.

10. Blom M, Kievit W, Fransen J, et al. The reason for discontinuation of the first tumor necrosis factor (TNF) blocking agent does not influence the effect of a second TNF blocking agent in patients with rheumatoid arthritis. J Rheumatol. 2009;36(10):2171-77.

11. Wolf D, Skup M, Yang H, et al. Clinical outcomes associated with switching or discontinuation from anti-TNF inhibitors for nonmedical reasons. Clin Ther. 2017:39(4):849-62.e6.

12. Liu Y, Skup M, Lin J, Chao J. Impact of non-medical switching on healthcare costs: a claims database analysis. Value Health. 2015;18(3):A252

13. Signorovitch J, Bao Y, Samuelson T, Mulani PM. Switching from adalimumab to other disease-modifying antirheumatic drugs without apparent medical reasons in rheumatoid arthritis: impact on health care service use [abstract 1395]. Ann Rheum Dis. 2013;71(Suppl 3):717.

14. Wolf D, Skup M, Yang H, et al. Clinical outcomes associated with switching or discontinuation of anti-TNF inhibitors for non-medical reasons Clin Ther. 2017:39(4):849-62.e6.

15. Gibofsky A, Skup M, Mittal M, et al. Effects of non-medical switching on outcomes among patients prescribed tumor necrosis factor inhibitors. Curr Med Res Opin. 2017;33(11):1945-53.

16. Curtis JR, Baddley JW, Yang S, et al. Derivation and preliminary validation of an administrative claims-based algorithm for the effectiveness of medications for rheumatoid arthritis. Arthritis Res Ther. 2011:13(5):R155. 
17. Bonafede M, Johnson BH, Tang DH, Shah N, Harrison DJ, Collier DH. Etanercept-methotrexate combination therapy initiators have greater adherence and persistence than triple therapy initiators with rheumatoid arthritis. Arthritis Care Res (Hoboken). 2015;67(12):1656-63.

18. Baser O, Ganguli A, Roy S, Xie L, Cifaldi M. Impact of switching from an initial tumor necrosis factor inhibitor on health care resource utilization and costs among patients with rheumatoid arthritis. Clin Ther. 2015;37(7):1454-65.
19. Gu T, Mutebi A, Stolshek BS, Tan H. Cost of biologic treatment persistence or switching in rheumatoid arthritis. Am J Manag Care. 2018;24(8 Spec No.):SP338-45.

20. Dunn A, Grosse SD, Zuvekas SH. Adjusting health expenditures for inflation: a review of measures for health services research in the United States. Health Serv Res. 2018;53(1):175-96. 


\section{APPENDIX A Study Design}

A. Study Requirements

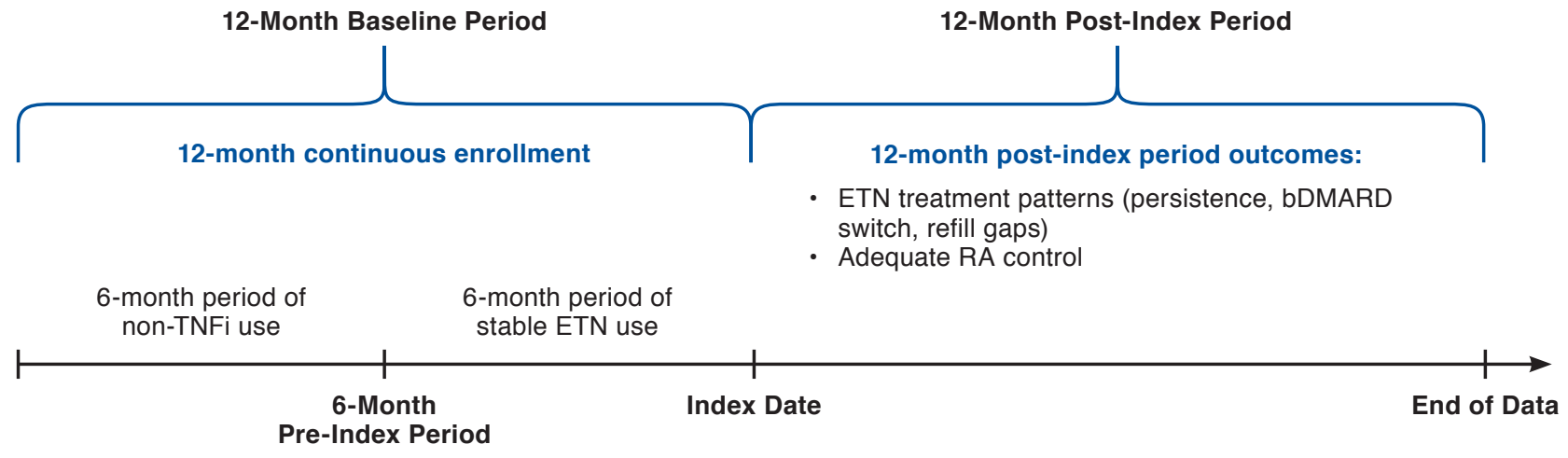

B. Study Requirements When Patient Had a Post-Index Treatment Change

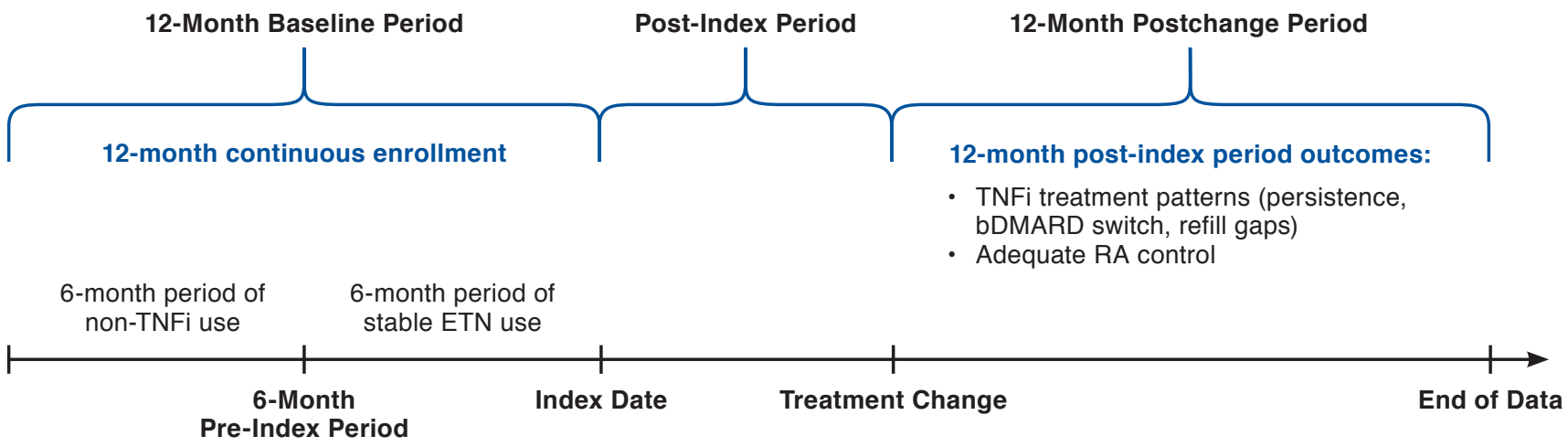

bDMARD = biologic disease-modifying antirheumatic drug; ETN = etanercept; RA = rheumatoid arthritis; TNFi= tumor necrosis factor inhibitor. 


\section{APPENDIX B Patient Selection}

Patients in the MarketScan Commercial Database with at least 1 pharmacy or medical claim for 1 of the following anti-TNF biologics: adalimumab, certolizumab pegol, etanercept, golimumab, or infliximab between January 1, 2013, and December 31, 2015

With at least 6 months of anti-TNF biologic use (with a gap of $<45$ days) between January 2, 2013, and December 31, 2015

Index date =day after the end date of 6 months of anti-TNF biologic persistent use

With at least 6 months of continuous enrollment with medical and pharmacy benefits before the index date

(i.e., anti-TNF biologic persistent-use period)

With at least 6 months of continuous enrollment with medical and pharmacy benefits before the stable antibiologic use period (i.e., prebiologic period)

No pharmacy or medical claims for anti-TNF biologics during the 6 months before the anti-TNF biologic persistent-use period

With at least 12 months of continuous enrollment after index date (post-index period)

With at least 1 inpatient or outpatient nondiagnostic medical claim with a diagnosis for rheumatoid arthritis in any position during the 12-month baseline period

Aged 18-64 years at index

$n=6,808$

No nondiagnostic claims with ICD-9/10-CM diagnosis codes for juvenile idiopathic arthritis, psoriasis, psoriatic arthritis, ankylosing spondylitis, Crohn disease, or ulcerative colitis in any position at any time during the study period

$n=5,149$
$\mathrm{N}=191,826$

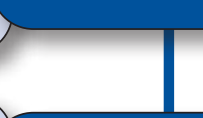

$n=119,797$

$n=80,583$

$n=26,548$

$n=19,779$

$n=6,887$
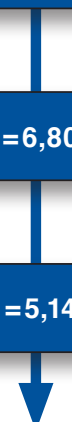

6 months stable etanercept use $n=1,970$

ICD-9/10-CM = International Classification of Diseases, Ninth/Tenth Revision, Clinical Modification; TNF = tumor necrosis factor. 